\title{
Traceable measurements of the activity concentration in air
}

\author{
Annette Paul and Anja Honig \\ Physikalisch-Technische Bundesanstalt (PTB), Bundesallee 100, D-38116 \\ Braunschweig
}

Doris Forkel-Wirth, André Mueller and Alicia Marcos

CERN/TIS/RP, CH-1211 Geneva 23

\begin{abstract}
The nuclear reactions induced by high energetic protons in heavy targets such as $\mathrm{UC}_{2}$ and $\mathrm{ThC}$ cause a particular, complex radiation protection task at facilities like ISOLDE: the measurement of a mixture of different isotopes of the radioactive noble gas radon and the radon progenies in air. The knowledge of their respective activity concentration is fundamental for exposure assessments.

Due to the complex mixture of activity concentrations in air, its precise determination is quite difficult. Therefore, a new procedure for taking reference samples was developed and implemented for the traceable measurement of the activity concentration of radioactive ions (e.g. radon progenies) in air. This technique is combined by measuring $\alpha$-particles with a multi-wire ionization chamber for the parallel on-line determination of the activity concentration of different radon isotopes.
\end{abstract}

PACS: 28.41.Te, 29.25.Rm, 29.30.h

Keywords: ISOLDE, $\alpha-$ and $\gamma$-spectrometry, radon and radon progenies

\section{Introduction}

ISOLDE is an on-line mass separator designed for the production of radioactive ion beams. The radioactive isotopes are produced via spallation reactions induced by the bombardment of thick targets with high energetic protons (1 or $1.4 \mathrm{GeV}$ ). Presently, up to 600 different isotopes from 60 different targetion source combinations can be provided to ISOLDE users. The targets range from light materials like $\mathrm{SiC}$ or $\mathrm{CaO}$ to heavy ones like $\mathrm{UC}_{2}$ or ThC. By irradiation of $\mathrm{UC}_{2}$ or $\mathrm{ThC}$ with protons, the whole range of radon isotopes as well as their parent isotopes are produced aside from others. Thus, at ISOLDE,

Preprint submitted to Elsevier Preprint $\quad 5$ September 2000 
two situations have to be considered. During or shortly after a $\mathrm{UC}_{2}$ or $\mathrm{ThC}$ run, the emanation of the directly produced gaseous activity has to be taken into account in addition to the permanent emanation from the parent isotopes implanted into the beam line system during former runs. After an appropriate decay time only the emanation from the implanted parent isotopes is significant [1].

The activity emitted, for example, by ventilation systems is measured by samples normally taken on active charcoal and glass fibre filters. Since the collection efficiencies for these sample materials are not very well known and data for specific isotopic collection efficiencies are not available, this measurement involves quite large systematic uncertainties. Moreover, this concept does not work for radon isotopes at all.

In the ISOLDE ventilation system a Lucas cell is implemented to check for the total $\alpha$-activity released into air. However, the assignment of the counted $\alpha$-particles to the isotopes they are emitted from is rather difficult and time consuming. This work presents a new concept for the determination of the activity concentration of different radon and radon progenies including collection efficiencies, as well as basic equations [2] and first results obtained at the switch yard of ISOLDE 2. By the implementation of a well-defined flow, the measurement of the time for taking the reference sample and the determination of the activity by $\alpha$ - and/or $\gamma$-spectrometry, the activity concentration of the progenies is established on the basis of new accurate collection efficiencies. Volume flow, time, and activity measurement are implemented in such a way that they are traceable to national or international standards [3]. Furthermore, a concept for the parallel on-line measurement of different radon isotopes is added [4,5], since the noble gases cannot be collected on filters. The correlation of the radon activity concentration and the progeny activity concentration in air results the equilibrium factor and, thus, accurate values for exposure calculations.

\section{Concept, definitions and basic equations}

The conventional 'radon problem' of radiation protection is due to the shortlived progenies of ${ }^{222} \mathrm{Rn}$ : their activity concentration in air is responsible for about $30 \%$ of the whole human exposure to ionizing radiation. In radiation protection, the exposure caused by ${ }^{222}$ Radon progenies is often estimated on the basis of a measurement of the radon activity concentration $C\left({ }^{222} \mathrm{Rn}\right)$ (probably with low-cost, easy-to-handle passive radon devices $\left.[6,7]\right)$. Although in radioactive equilibrium the activities of the short-lived radon progenies are equal to the ${ }^{222} \mathrm{Rn}$ activity, the activity concentrations in air are often found to be $C\left({ }^{222} \mathrm{Rn}\right) \geq C\left({ }^{A} \mathrm{RnP}\right)$, where $\mathrm{A}$ is the mass number of the short-lived radon progeny $\mathrm{RnP}\left({ }^{218} \mathrm{Po},{ }^{214} \mathrm{~Pb},{ }^{214} \mathrm{Bi},{ }^{214} \mathrm{Po}\right)$. This is caused 
by the plate-out effect: the attachment of radon progenies to surfaces, thus reducing their fraction in air. We define the degree of equilibrium in air by $k_{\mathrm{AnP}}=C\left({ }^{\mathrm{A}} \mathrm{RnP}\right) \cdot C\left({ }^{222} \mathrm{Rn}\right)^{-1}$, which requires the measurement of both, the radon and the radon progeny activity concentration. Note that this definition is valid for the state of both activity equilibrium and non-equilibrium. To avoid such quite extensive and expensive measurements, for the purposes of radiation protection, the so-called equilibrium factor $F$ was introduced by ICRP [8]. The basic idea for this equilibrium factor is to define a value as a relative measure of the amount of activity concentration of short-lived radon progenies in air under all conditions. Taking all short-lived progenies into account (and this in a radiologically sensible way with regard to a normalized potential alpha energy concentration), it has to be a weighted average over the degree of equilibrium in air

$$
F=\sum_{\mathrm{A}_{\mathrm{RnP}}} K_{\mathrm{A}_{\mathrm{RnP}}} \cdot k_{\mathrm{A} \mathrm{RnP}} \quad \text { with } \quad K_{\mathrm{A} \mathrm{RnP}}=\frac{\frac{E_{\mathrm{p},{ }^{\mathrm{A}} \mathrm{RnP}}}{\lambda_{\mathrm{A} R n P}}}{\sum_{\mathrm{A}_{\mathrm{RnP}}} \frac{E_{\mathrm{p},{ }^{\mathrm{A}} \mathrm{RnP}}}{\lambda_{\mathrm{A} R n P}}}
$$

In this notation, $E_{\mathrm{p},{ }^{\mathrm{A}} \mathrm{RnP}}$ is the potential alpha energy, i. e. the sum of alpha energies emitted by this nuclide and its decay products during its decay up to ${ }^{210} \mathrm{~Pb}\left({ }^{210} \mathrm{~Pb}\right.$ is already excluded $)$, and $\lambda_{\mathrm{A}} \mathrm{RnP}$ is its decay constant.

This terminology normally is exclusively used for ${ }^{222} \mathrm{Rn}$, therefore, in the case of the complex radon atmosphere at ISOLDE, these definitions have to be extended for different radon isotopes ${ }^{A_{0}} \mathrm{Rn}$ with the corresponding $K_{\mathrm{A}}^{\mathrm{A}_{0} \mathrm{Rn}}$ given in tab. 1:

$$
k_{\mathrm{A} R n}^{\mathrm{A}_{0} \mathrm{Rn}}=\frac{C\left({ }^{\mathrm{A}} \mathrm{RnP}\right)}{C\left({ }^{\mathrm{A}_{0}} \mathrm{Rn}\right)} \quad \text { and } \quad F\left({ }^{\mathrm{A}_{0}} R n\right)=\sum_{\mathrm{A}_{\mathrm{RnP}}} K_{\mathrm{A} R \mathrm{P}}^{\mathrm{A}_{0} \mathrm{Rn}} \cdot k_{\mathrm{A} R \mathrm{RP}}^{\mathrm{A}_{0} \mathrm{Rn}}
$$

\begin{tabular}{|c|c|c|c|c|}
\hline${ }^{A_{0}} \mathrm{Rn}$ & \multicolumn{4}{|c|}{$K_{\mathrm{A} \mathrm{RnP}}^{\mathrm{A}_{0} \mathrm{P}}$} \\
\hline \hline${ }^{219} \mathrm{Rn}-$ & $K_{215} \mathrm{Po}$ & $K_{211} \mathrm{~Pb}$ & $K_{211} \mathrm{Bi}$ & $K_{211} \mathrm{Tl}$ \\
decay chain & $1.64(1) \cdot 10^{-9}$ & $0.944(8)$ & $0.0559(6)$ & 0 \\
\hline${ }^{220} \mathrm{Rn}-$ & $K_{216} \mathrm{Po}$ & $K_{212} \mathrm{~Pb}$ & $K_{212} \mathrm{Bi}$ & $K_{212} \mathrm{Po}$ \\
decay chain & $6.45(9) \cdot 10^{-6}$ & $0.913(1)$ & $0.0866(1)$ & $8.01(6) \cdot 10^{-12}$ \\
\hline${ }^{222} \mathrm{Rn}-$ & $K_{218} \mathrm{Po}$ & $K_{214} \mathrm{~Pb}$ & $K_{214} \mathrm{Bi}$ & $K_{214} \mathrm{Po}$ \\
decay chain & $0.106(2)$ & $0.513(20)$ & $0.381(10)$ & $5.2(1) \cdot 10^{-8}$ \\
\hline
\end{tabular}

Table 1

The corresponding quantities $K_{\mathrm{A} R \mathrm{R}}^{\mathrm{A}_{0} \mathrm{P}}$ for three radon decay chains: ${ }^{219} \mathrm{Rn},{ }^{220} \mathrm{Rn}$ and ${ }^{222} \mathrm{Rn}$. Branchings below $0.3 \%$ (for example leading to ${ }^{211} \mathrm{Po}$ ) are not included. Nuclear data is taken from [9]. 
In this formal way it is possible to define an equilibrium factor $F\left({ }^{\mathrm{A}_{0}} \mathrm{Rn}\right)$ for each radon decay chain. It is an open question whether it is reasonable to proceed in this way, since the basic idea of the concept of an equilibrium factor is, that an activity equilibrium is possible, which is, according to the involved half-lives, possible only for ${ }^{222} \mathrm{Rn}$. Since the use of the quantity 'equilibrium factor $F^{\prime}$ is well-established for ${ }^{222} \mathrm{Rn}$ and an extension for ${ }^{220} \mathrm{Rn}$ (thoron) [8] (following probably the line of argument of a constant exhalation source, leading to an equilibrium state) was defined, this terminology is further extended in this work.

Anyway, $k_{\mathrm{A} R \mathrm{R}}^{\mathrm{A}_{0} \mathrm{Rn}}$ is probably the most reliable and sensitive quantity to describe a radon atmosphere. This involves a set-up for the measurement of the activity concentration of different radon isotopes in air, as well as one for the collection and measurement of their progenies.

\section{Experimental set-up for the activity concentration of radon}

The determination of the activity concentration of different radon isotopes is implemented via on-line $\alpha$-spectrometry in air by a multi-wire pulse ionization chamber.

For the measurement of the noble gas radon, $\alpha$-spectrometry has to be performed in air at normal pressure. It is, therefore, not possible to use entrance windows, gas amplification or some kind of counting gas. Also, electron collection (because electrons are captured by electronegative gases, e. g. oxygen) cannot be used. Since the electrons are captured, only ions are suitable for collection in pulses for the purpose of spectrometry. The gas amplification has to be one, since the chamber must be run in a spectrometry mode and not for counting. The energy of the $\alpha$-particle is determined by the number of ions produced during its passage through air. The ions are collected by the multiwire array which is at high electric potential. This results in a small current pulse. Due to ion collection, the pulse is quite long and of low amplitude of about $1 \mathrm{fA}$ in $30 \mathrm{~ms}[4,5]$ for an $\alpha$-energy of about $6 \mathrm{MeV}$.

The multi-wire chamber used here was compared to the instruments of the German radon reference chamber in an activity range from 10 to $10^{4} \mathrm{~Bq} / \mathrm{m}^{3}$ and calibrated at the point of $3700(90) \mathrm{Bq} / \mathrm{m}^{3}$ against a radon standard source. All these measurements were based on ${ }^{222} \mathrm{Rn}$ activity concentration, only. Nevertheless, considering the principle of the multi-wire ionization chamber, it is justified to adopt the same method of analysis, including active volume and calibration factor, for the activity concentration of other radon isotopes. The energy calibration is based on ${ }^{222} \mathrm{Rn},{ }^{218} \mathrm{Po}$ and ${ }^{214} \mathrm{Po}$ and can be used to identify other radon isotopes. Since the radon progenies cannot enter the multiwire ionization chamber (air filter system), only those produced in the chamber are measured. The progenies which are produced there, attach to wires 
and cannot leave the chamber. Under stable conditions, this induces a fixed ratio between each radon isotope and each of its progenies. This 'fixed-ratio approximation' considerably reduces the parameters for fitting the $\alpha$-spectra. Fig. 1 shows an example for such an analysis. Only the activity concentration of ${ }^{219} \mathrm{Rn},{ }^{220} \mathrm{Rn}$ (and ${ }^{212} \mathrm{Po}$ due to its long half-life) and ${ }^{222} \mathrm{Rn}$ are free parameters. The FWHM is chosen to be the same for all peaks, the position of the peaks is given by a linear energy calibration based on literature data and the background is adjusted according to the calibration data.

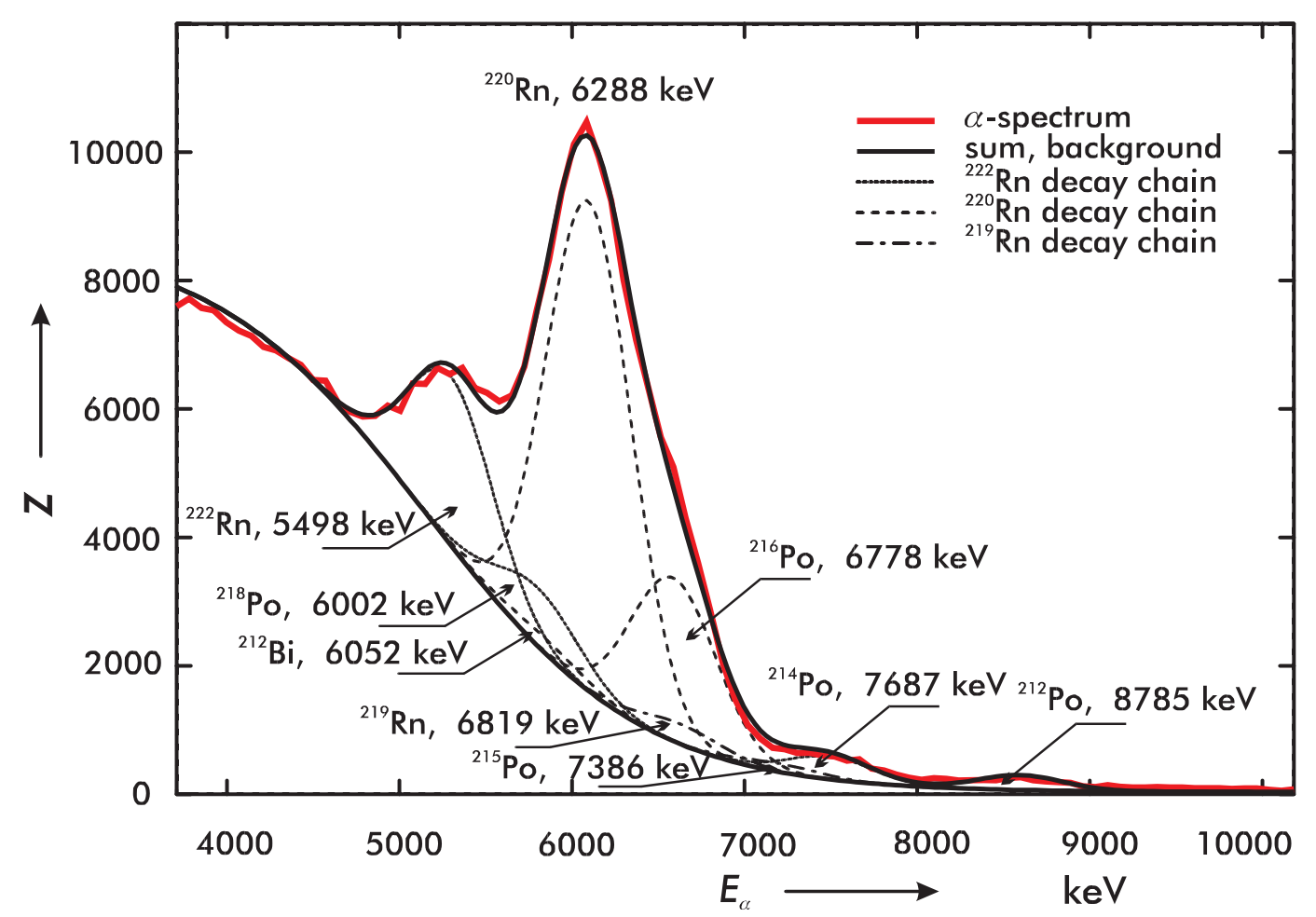

Fig. 1. Radon and radon progenies of three decay chains measured by $\alpha$-spectrometry in air with the multi-wire ionization chamber.

It has to be noted that the activity concentration measured with the multiwire ionization chamber is the activity concentration inside the chamber itself and not inside the system to which it is connected. Due to the short half-life of ${ }^{219} \mathrm{Rn}$ and ${ }^{220} \mathrm{Rn}$, corrections for the transport time are necessary. These corrections are performed on the basis of known tube length and volume flow. In case of the very short-lived radon isotopes, the uncertainty of these corrections can add significantly to the total uncertainty of the resulting activity concentration.

After an on-line measurement is finished, the subsequent decay of the progenies deposited inside the multi-wire ionization chamber can be observed. This can be done without any interference from radon isotopes, if the chamber is ventilated by air of low activity. Outside air is often sufficient, most of the technical airs for industrial uses have even lower activities. 


\section{Experimental set-up for the activity concentration of radon pro- genies}

The radon progenies are collected on so-called reference samples (by a welldefined flow during a well-defined collection time) and measured offline via $\alpha$ - and/or $\gamma$-spectrometry. A standard set-up consisting of a surface barrier detector and a high-purity germanium detector is sufficient. The dimensions of the reference samples are chosen to be identical to those of the calibration sources. As a result, no corrections for the geometry of the measurement are necessary and, therefore, the dimensions do not add any uncertainty to the resulting activity concentration. There are two different types of reference samples: a stainless steel screen with parallel wires of $100 \mu m$ distance to each other (diameter of stainless steel wires: $63 \mu \mathrm{m}$ ) and a thin glass fibre filter. Both have a diameter of $20 \mathrm{~mm}$ and are fixed in an aluminum ring. The outerside diameter of the reference samples is $30 \mathrm{~mm}$.

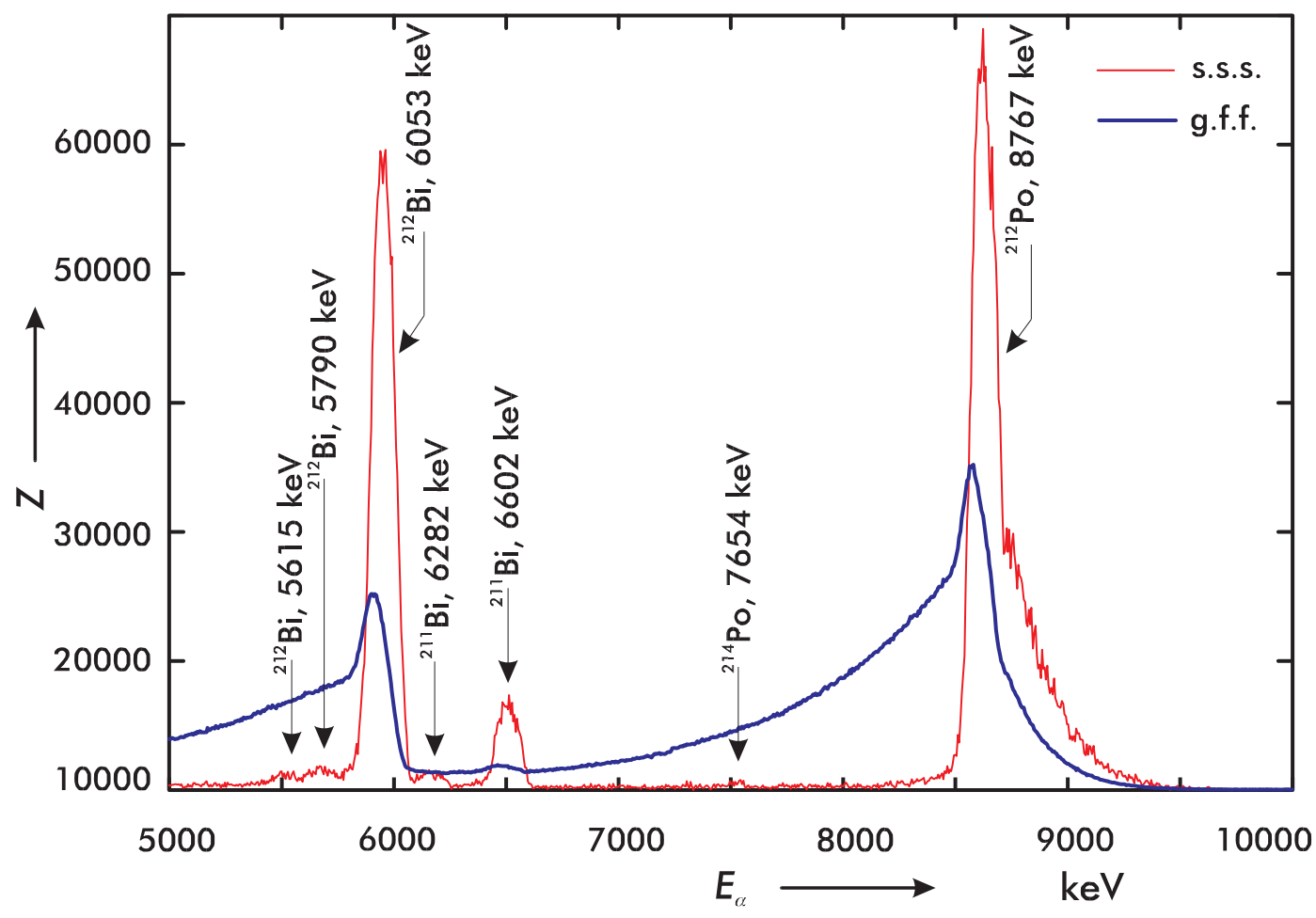

Fig. 2. Comparison of the $\alpha$-spectra of a sample on a stainless steel screen (s.s.s.) and a glass fibre filter (g.f.f.). The peak height of the $\alpha$-spectra of a sample on the stainless steel screen is normalized according to the collection efficiency to show details. The high-energy tail of ${ }^{212} \mathrm{Po}$ might be due to coincidence summing of $\alpha$-particles and electrons emitted from ${ }^{212} \mathrm{Po}$ and ${ }^{212} \mathrm{Bi}$, respectively.

For short-lived ${ }^{222} \mathrm{Rn}$-progenies systematic investigations $[2,10]$ of the capability of the reference samples in a well-defined air flow showed high and reproducible collection efficiencies according to the state of attachment of the radioactive ion. Of the so-called 'unattached fraction' (progenies which are not 
attached to aerosols) 95.2(10) \% can be collected on the stainless steel screen, while of the 'attached fraction' (progenies which are attached to aerosols) only $1.5(5) \%$ is collected. On the other hand, 98.5(15) \% of the unattached fraction and the $98.9(7) \%$ of the attached fraction is colleced on the glass fibre filter. Though an extended validation for these values was performed for the $219,220,222$ Rn-progenies (heavy metal ions), a further adjustment or validation for the other radioactive ions (for example iodine) may be necessary.

The isotopic collection efficiencies $S_{s, f}$ are determined by placing two reference samples of the same type, one before each other, in the air flow. This is achieved by a tight sample tube, which ensures that the flow through each sample is the same. This determination of collection efficiencies is independent of the detector efficiency, the geometry of the measurement, the transition probability of the nuclide and, for most of the time, of its half-life. It must only be assumed that the collection efficiencies of the two reference samples are equal. Nevertheless for short-lived isotopes corrections according to the transport time between the two reference samples have to be applied. It has to be noted that the collection efficiencies $S_{s}$ for the stainless steel screens are strictly dependent on the state of attachment of the isotopes, and therefore should not be used for activity calculations. A high value $S_{s}$ is caused by a large unattached fraction, only. Since in closed systems the conditions regarding the aerosol concentration (and therefore for the state of attachment) do to not vary considerably, it can be justified to use these values as well. Moreover, the stainless steel screen provides almost ideal samples for $\alpha$-spectrometry (s. fig. 2), because the ions are deposited on the surface and not in a matrix (like filters, even the very thin glass fibre filters). The $\alpha$-spectra of the stainless steel screen samples therefore have the best resolution. Nevertheless, all calculations of activity concentrations have to rely on the $S_{f}$ values, since these values are in the range of uncertainty independent from the state of attachment.

\section{Results}

The test and first application of this method took place at the switch yard of ISOLDE 2. This device served for many years until the SC-ISOLDE facility was closed down in 1990 . The radon activity concentration observed in this vacuum chamber originates from the radon mother isotopes that were implanted into the walls of the chamber until end of 1990. The set-up includes the sample tube for taking reference samples (connected to a flow meter and pump in a fixed set-up named LPH) and the multi-wire ionization chamber. The activity concentrations of the radon progenies are thus determined offline by $\alpha$ - and $\gamma$-spectrometry, while the activity concentrations of the different radon isotopes are determined on-line and in parallel. 


\begin{tabular}{|c||c|c|c|c||c|}
\hline$C\left({ }^{219} \mathrm{Rn}\right)$ & $C\left({ }^{215} \mathrm{Po}\right)$ & $C\left({ }^{211} \mathrm{~Pb}\right)$ & $C\left({ }^{211} \mathrm{Bi}\right)$ & $C\left({ }^{207} \mathrm{Tl}\right)$ & $F\left({ }^{219} \mathrm{Rn}\right)$ \\
$\mathrm{kBq} / \mathrm{m}^{3}$ & $\mathrm{kBq} / \mathrm{m}^{3}$ & $\mathrm{kBq} / \mathrm{m}^{3}$ & $\mathrm{kBq} / \mathrm{m}^{3}$ & $\mathrm{kBq} / \mathrm{m}^{3}$ & \\
\hline $94(14)$ & - & $23(2)$ & eq. $C\left({ }^{211} \mathrm{~Pb}\right)$ & eq. $C\left({ }^{211} \mathrm{~Pb}\right)$ & $0.24(4)$ \\
\hline \hline$C\left({ }^{220} \mathrm{Rn}\right)$ & $C\left({ }^{216} \mathrm{Po}\right)$ & $C\left({ }^{212} \mathrm{~Pb}\right)$ & $C\left({ }^{212} \mathrm{Bi}\right)$ & $C\left({ }^{212} \mathrm{Po}\right)$ & $F\left({ }^{220} \mathrm{Rn}\right)$ \\
$\mathrm{kBq} / \mathrm{m}^{3}$ & $\mathrm{kBq} / \mathrm{m}^{3}$ & $\mathrm{kBq} / \mathrm{m}^{3}$ & $\mathrm{kBq} / \mathrm{m}^{3}$ & $\mathrm{kBq} / \mathrm{m}^{3}$ & \\
\hline $171(26)$ & - & $51(2)$ & $89(32)$ & eq. $C\left({ }^{212} \mathrm{Bi}\right)$ & $0.32(5)$ \\
\hline \hline$C\left({ }^{222} \mathrm{Rn}\right)$ & $C\left({ }^{218} \mathrm{Po}\right)$ & $C\left({ }^{214} \mathrm{~Pb}\right)$ & $C\left({ }^{214} \mathrm{Bi}\right)$ & $C\left({ }^{214} \mathrm{Po}\right)$ & $F\left({ }^{222} \mathrm{Rn}\right)$ \\
$\mathrm{kBq} / \mathrm{m}^{3}$ & $\mathrm{kBq} / \mathrm{m}^{3}$ & $\mathrm{kBq} / \mathrm{m}^{3}$ & $\mathrm{kBq} / \mathrm{m}^{3}$ & $\mathrm{kBq} / \mathrm{m}^{3}$ & \\
\hline$\leq 2$ & $0.6(6)$ & $0.7(1)$ & $0.7(2)$ & eq. $C\left({ }^{214} \mathrm{Bi}\right)$ & $\geq 0.3$ \\
\hline
\end{tabular}

Table 2

Activity concentration in air and equilibrium factors (s. equation 2) determined at the switch yard of ISOLDE 2. The stated uncertainties are standard uncertainties. An activity equilibirium on the sample is expressed by eq. $C\left({ }^{\mathrm{A}} \mathrm{RNP}\right)$.

During all measurements, only activity from or subsequent to the decay of the radon isotopes is detected. It can be concluded that the transport of the implanted activity to the air is caused alone by the diffusion of the noble gas radon. The radon progenies are produced as heavy metal ions and therefore attach themselves to the surfaces. An activity equilibrium in air for radon and its progenies is not reached. The result determined is given in the order of the decay chain, in tab. 2. The values for $C\left({ }^{219} \mathrm{Rn}\right)$ are corrected for time it takes to transport the air into the ionization chamber. Due to the transport time (some minutes) of the reference targets from the point where the sample has been taken to the detectors, $C\left({ }^{215} \mathrm{Po}\right)$ and $C\left({ }^{216} \mathrm{Po}\right)$ were not detectable due to short half-lives. Furthermore, short-lived progenies collected on the reference samples are often already found in activity equilibrium with their more longlived parents (according to the branching ratio, if one exist). They are marked with eq. $C\left({ }^{\mathrm{A}} \mathrm{RNP}\right)$ to express this fact. If they are not in equilibrium but very close to it, large uncertainties are provoked by the subtraction of the activity of the parent nuclide. Finally, the long-lived progenies of ${ }^{222} \mathrm{Rn}$ are not included in this collection.

The uncertainties assigned to the radon activity concentrations are large in this example due to the high count rate corresponding to a large dead time correction. Nevertheless, this measurement demonstrates that activity concentrations beyond $100 \mathrm{kBq} / \mathrm{m}^{3}$ can be measured with multi-wire ionization chambers. The ratio $k_{\mathrm{A} n \mathrm{R}}^{\mathrm{A}_{0} \mathrm{Rn}}$ (s. equation 2) of activity concentration of progenies in air to the corresponding radon activity concentration is, in the range of uncertainty, the same: 0.24 (4) for ${ }^{219} \mathrm{Rn}, 0.30(5)$ and $0.52(20)$ for ${ }^{220} \mathrm{Rn}$ and $\geq 0.3$ for ${ }^{222} \mathrm{Rn}$. This is a good indication (even more sensitive than the equilibrium factor $F$ ) of a stable system with high activity concentrations. Consequently, a survey via progeny measurement is probably the best choice 
for routine measurements.

\section{Conclusions}

The determination of the activity concentration in complex radon atmospheres is a new topic, both for metrology and radiation protection. Further studies are necessary to develop calibration facilities, to provide reference atmospheres and to implement traceable standard procedures for radiation protection and radiation protection measurements.

First measurements at different ISOLDE systems showed the need for a more sophisticated approach for the determination of the activity concentration, if implantation of radon parent nuclides is involved. Comparisons furnished results differently up to a factor of 10 , due to the complexity of the decay chains, which cannot be covered by standard instrumentation and analysis. Therefore, the method of taking reference samples was developed and implemented, as well as the modification of the $\alpha$-spectra analysis for the multi-wire ionization chamber. This offers the possibility to determine both, the radon and the radon progeny activity concentration in air. The results obtained by these two methods are consistent and the calibration for the progeny activity concentration is already traceable. For the radon activity concentration a validation via a comparison and one-point calibration exists. Nevertheless it has to be noted that the measurement and analysis procedures for the two systems are more complex than typical routine procedures in radiation protection services.

Within the scope of the cooperation between CERN and PTB, a study of the even more complex radon mixtures produced by ISOLDE in operation will be considered as well. The characterization of such an atmosphere ventilated towards the exterior (including the whole range of different radon isotopes in the case of heavy targets), is a pending question to be solved. Presently, the problem is circumvented by a strong overestimation of the radiation doses possibly involved. Our project could be a first step, towards joint efforts together with other accelerator groups with similar radiation protection problems.

\section{Acknowledgements}

We would like to thank Dr. Stefan Röttger (PTB, Braunschweig) for his expertise in the optimization of the multi-wire ionization chamber and Prof. Dr. Uwe Keyser (PTB/Q.2) for his help in establishing the cooperation.

Furthermore, we would like to express our gratitude to Hubert Müller for the fast and excellent construction of the sample tube LPH ('Le Petit Hubert'). 
Moreover, we owe thanks to the technicians running and maintaining the spectrometry and assuring the radiation protection at the experimental sites, Guy Roubaud, Jacques Wolf and Florence Pirotte for their valuable support. Finally we would like to thank Pavol Vojtyla for the supply of environmental data about the ISOLDE ventilation system.

\section{References}

[1] M. Durcik, F. Havlik. Experimental study of radon and thoron diffusion through barriers. Journal of Radioanalytical and Nuclear Chemistry, 209:307313 (1996).

[2] A. Paul, S. Röttger, A. Honig, T. Sulima, A. Buchholz and U. Keyser. Measurement of short-lived radon progenies by $\alpha \gamma$-spectrometry at the radon reference chamber. Nuclear Instruments and Methods in Physics Research, A434:303-312 (1999).

[3] T. Quinn. Basic metrological elements common to traceability of any measurement. Ad-Hoc Working Group on the Avogadro Constant of the CCM, IRMM, Geel, 05.11.1997.

[4] Gammadata Mätteknik AB, P.0.Box 15120, S-75015 Uppsala, Sweden. Atmos 12 DPX, Radon gas monitor for indoor measurement (1994).

[5] S. Röttger, A. Paul, A. Honig and U. Keyser. Vieldraht-ImpulsIonisationskammern zur Präzisionsmessung der Radon-Aktivitätskonzentration in Luft. PTB-Report PTB-PG-10, Physikalisch-Technische Bundesanstalt (1998).

[6] Deutsches Institut für Normung e. V. Passive Radonmessungen, Teil 1: Kernspurmeßverfahren. DIN 25706-1, Beuth Verlag GmbH, 10772 Berlin (1994).

[7] Deutsches Institut für Normung e. V. Passive Radonmessungen, Teil 2: Aktivkohlemeßverfahren. DIN 25706-2, Beuth Verlag GmbH, 10772 Berlin (1994).

[8] International Commission on Radiological Protection. ICRP Publication 50. Ann. of ICRP, 17:1-60 (1987).

[9] Nuclear Data. Decay Radiations. National Nuclear Data Center, Brookhaven National Laboratory Upton, N.Y., USA (1998).

[10] A. Paul, A. Honig, S. Röttger and U. Keyser. Measurement of radon and radon progenies at the German radon reference chamber. Appl. Radiat. Isot., 52/3-4:369-375 (2000). 